\title{
Linear Response and Fluctuation Theorems for Nonstationary Stochastic Processes ${ }^{\star}$
}

\author{
P. Hänggi and $H$. Thomas \\ Institut für Physik, Universität Basel, Switzerland
}

Received August 5, accepted August 22, 1975

\begin{abstract}
Linear response theory is developed for nonstationary Markov processes. A generalized fluctuation theorem is derived which relates the response function to a correlation of fluctuations of the unperturbed nonstationary process. It is shown that it reduces to an ordinary fluctuation theorem relating the response function to the two-point correlation between fluctuations of state variables in the case of a Gaussian distribution function. The results are illustrated by explicit calculation for the class of nonstationary linear Fokker-Planck processes.
\end{abstract}

\section{Introduction}

Most of the work on stochastic processes has been concerned with stationary processes occurring in systems under time-independent external conditions [1]. The statistical properties of such systems are independent of time, after the transients resulting from the preparation of the system have died out. But there exist many systems which are driven by external forces varying in time giving rise to nonstationary processes. Examples are conductors and plasmas in time-dependent electric and magnetic fields, lasers driven in a time-dependent way, chemical reactions fed by time-dependent fluxes of its components, further biological and sociological systems in a changing environment. Also other processes such as speech signals, pressure distribution in turbulent flow, and earth-quake disturbances may perhaps be more adequately described in terms of nonstationary processes.

In this paper, we are concerned with the (non-perturbative) case of large time-dependent forces, the effect of which cannot be described by a low-order perturbation of a stationary process. We study the relation between the fluctuations in such a system and its linear response to an additional dynamic perturbation. Fluctuation theorems connecting the linear response with the correlations of the fluctuations in

\footnotetext{
* Supported by Schweizerischer Nationalfonds.
}

the unperturbed state play an important role in the statistical mechanics of systems in thermodynamic equilibrium (here one has the famous fluctuationdissipation theorem $[2,3]$ ), in the thermodynamics of irreversible processes $[2,4]$, as well as in the theory of stationary stochastic processes [5-9]. The existence of a fluctuation theorem can therefore be expected to be of significance also in the theory of nonstationary processes. It simplifies, for instance, considerably the renormalized perturbation theory for classical processes [10]. In the present paper, we construct fluctuation theorems for the large class of nonstationary Markov processes with Gaussian distributions which are perturbed by a gradient term.

In Section 2, we summarize some general properties of nonstationary Markov processes, and introduce the correlation matrix of the fluctuations. The linear response theory for such processes is developed in Section 3 and is applied to Fokker-Planck processes. We derive a generalized fluctuation theorem which reduces to an ordinary fluctuation theorem for the case of a Gaussian distribution and a gradient-type perturbation. The theory is illustrated in Section 4 by explicit calculations for the specific class of "linear" Fokker-Planck processes which contains the GaussMarkov process [11] and the additive Fokker-Planck process [12]. The results obtained are discussed briefly in Section 5 . 


\section{Nonstationary Markov Processes}

We assume that the states of the system to be considered are described by sets of stochastic variables $\boldsymbol{x}=\left\{x_{1}, \ldots x_{n}\right\}$ forming the state space $\Sigma$. The statistical distribution at time $t$ is given by the probability density function $p(t)$ defined on $\Sigma$, such that the statistical expectation of any state function $\phi(t)$ at time $t$ is obtained as

$$
\langle\phi(\boldsymbol{x}(t), t)\rangle=\int p(\boldsymbol{x} t) \phi(\boldsymbol{x} t) d x .
$$

We consider a Markov process for which the rate of change of the probability density is completely determined by the probability density at the same time (no memory!):

$\frac{d p(t)}{d t}=\Gamma(t) p(t)$

("forward equation"). Here, $\Gamma(t)$ is the linear stochastic operator acting on the space $\Pi$ of probability density functions, and is - in contrast to stationary processes - assumed explicitely time-dependent. In general, $\Gamma(t)$ is an integral operator. In the case of a continuous Markov process, it reduces to the wellknown Fokker-Planck differential operator.

The formal solution of Eq. (2.2) can be written as a linear transformation $R$ of the space $\Pi$,

$p\left(t_{2}\right)=R\left(t_{2} \mid t_{1}\right) p\left(t_{1}\right), \quad\left(t_{2}>t_{1}\right)$

in terms of the time evolution operator

$R\left(t_{2} \mid t_{1}\right)=T \exp \left\{\int_{t_{1}}^{t_{2}} \Gamma(s) d s\right\}$

where $T$ is the time ordering operator. $R\left(t_{2} \mid t_{1}\right)$ is assumed nonsingular for $t_{2}$ and $t_{1}$ finite. The integral kernel $R\left(x t_{2} \mid y t_{1}\right)$ of the operator $R\left(t_{2}, t_{1}\right)$ has the significance of a transition probability density from state $\boldsymbol{y}$ at time $t_{1}$ to state $\boldsymbol{x}$ at time $t_{2}$, i.e. of a conditional probability density at time $t_{2}$ if the state at time $t_{1}$ is fixed at $\boldsymbol{y}$. As a consequence, the operator $R\left(t_{2} \mid t_{1}\right)$ must satisfy the Chapman-Kolmogorov equation [13],

$R\left(t_{2} \mid t_{1}\right)=R\left(t_{2} \mid t\right) R\left(t \mid t_{1}\right) \quad$ for $t_{2}>t>t_{1}$.

Further, if $R$ is "honest" [14],

$\int R\left(x t \mid y t_{1}\right) d x=1$,

indicating that no systems are "lost" from the statistical ensemble in the stochastic process.

From $R\left(t_{2} \mid t_{1}\right)$, joint probability densities can be constructed and time correlations can be calculated. The two-time distribution is given by

$p^{(2)}\left(\boldsymbol{x} t_{2}, \boldsymbol{y} t_{1}\right)=R\left(\boldsymbol{x} t_{2} \mid \boldsymbol{y} t_{1}\right) p\left(y t_{1}\right), \quad\left(t_{2}>t_{1}\right)$ and the correlation of two state functions $\phi\left(t_{2}\right)$ and $\psi\left(t_{1}\right)$ is calculated as

$\left\langle\phi\left(\boldsymbol{x}\left(t_{2}\right), t_{2}\right) \psi\left(\boldsymbol{x}\left(t_{1}\right), t_{1}\right)\right\rangle$

$=\iint R\left(\boldsymbol{x} t_{2} \mid \boldsymbol{y} t_{1}\right) p\left(\boldsymbol{y} t_{1}\right) \phi\left(x t_{2}\right) \psi\left(y t_{1}\right) d x d y$.

Of main importance is the correlation matrix of the fluctuations of the state variables,

$\boldsymbol{\xi}=\boldsymbol{x}-\langle\boldsymbol{x}(t)\rangle$

which is (for real variables) defined as

$\mathbf{S}\left(t_{2}, t_{1}\right)=\left\langle\xi\left(t_{2}\right) \xi\left(t_{1}\right)\right\rangle$

$=\left\langle\boldsymbol{x}\left(t_{2}\right) \boldsymbol{x}\left(t_{1}\right)\right\rangle-\left\langle\boldsymbol{x}\left(t_{2}\right)\right\rangle\left\langle\boldsymbol{x}\left(t_{1}\right)\right\rangle$.

$\mathbf{S}\left(t_{2}, t_{1}\right)$ is obviously positive semidefinite and satisfies

$\mathbf{S}\left(t_{2}, t_{1}\right)=\tilde{\mathbf{S}}\left(t_{1}, t_{2}\right)$

( $\tilde{\mathbf{S}}$ is the transpose of $\mathbf{S}$ ). Further properties of the correlation matrix are discussed in Refs. 15 and 16.

The time evolution operator $R\left(t \mid t_{0}\right)$ relates the probability density $p(t)$ at time $t$ to the initial probability density $p\left(t_{0}\right) \equiv p_{0}$ at the time $t_{0}$ of preparation of the system:

$p(t)=R\left(t \mid t_{0}\right) p_{0}$.

As a consequence, all expectations and correlations will in general depend on $p_{0}$. It is of interest to study under which conditions the statistical properties of the system become independent of the way of preparation, i.e. of the initial distribution $p_{0}$, in the course of time. One therefore needs the extension of the concept of ergodicity to the nonstationary case. A process is called ergodic if $R(t \mid-\infty)$ exists and represents a singular operator which maps the whole $\Pi$ space onto a single element $p^{\text {as }}(t) \in \Pi$ independent of $p_{0}$,

$R(\boldsymbol{x} t \mid \boldsymbol{y},-\infty)=p^{\text {as }}(\boldsymbol{x} t)$.

An ergodic process thus evolves to a given asymptotic distribution $p^{\text {as }}(t)$, independent of the way and time of preparation (asymptotic stability).

\section{Linear Response Theory}

\subsection{General Formalism}

We want to study the linear response of our system to an external perturbation. We assume that the process remains of Markov character in the presence of the perturbation, such that the perturbed system is described by a stochastic operator

$\hat{\Gamma}(t)=\Gamma(t)+\Gamma^{\mathrm{ext}}(t)$ 
which gives rise to the time evolution operator

$\hat{R}\left(t \mid t_{0}\right)=T \exp \left\{\int_{t_{0}}^{t}\left(\Gamma(s)+\Gamma^{\mathrm{ext}}(s)\right) d s\right\}$

The exponential cannot simply be split into a product because $\Gamma(t)$ and $\Gamma^{\text {ext }}(t)$ in general do not commute. By employing the disentangling techniques of Feynman [17] and observing the nonsingularity of the unperturbed time evolution operator $R(t \mid s)$, we can write

$\hat{R}\left(t \mid t_{0}\right)=R\left(t \mid t_{0}\right) T \exp \left\{\int_{t_{0}}^{t} \Gamma^{\prime \mathrm{ext}}(s) d s\right\}$

where

$\Gamma^{\text {ext }}(s)=R\left(s \mid t_{0}\right)^{-1} \Gamma^{\mathrm{ext}}(s) R\left(s \mid t_{0}\right)$.

Thus, to first order in $\Gamma^{\mathrm{ext}}(t)$, we obtain

$\hat{R}\left(t \mid t_{0}\right)=R\left(t \mid t_{0}\right)+\int_{t_{0}}^{t} R(t \mid s) \Gamma^{\mathrm{ext}}(s) R\left(s \mid t_{0}\right) d s$

where we have made use of the group property Eq. (2.5). From $\hat{R}\left(t \mid t_{0}\right)$, we can calculate the perturbed distribution

$$
\begin{aligned}
& \hat{p}(t)=\hat{R}\left(t \mid t_{0}\right) p_{0} \\
& =p(t)+\int_{t_{0}}^{t} R(t \mid s) \Gamma^{\mathrm{ext}}(s) p(s) d s .
\end{aligned}
$$

Here, we have assumed that the perturbation is applied after the system has been prepared at time $t_{0}$ in a given distribution $p_{0}$.

We write the perturbation $\Gamma^{\mathrm{ext}}(t)$ in terms of timedependent external forces $F_{i}(t)$

$\Gamma^{\mathrm{ext}}(t)=\sum_{i} F_{i}(t) \Omega_{i} \equiv \boldsymbol{F}(t) \cdot \boldsymbol{\Omega}$

where the $\Omega_{i}$ are linear operators acting on the state space $\Sigma$. The linear response tensor $\chi(t, s)$ is then defined by the relation of the response of the state variables

$$
\begin{aligned}
& \langle\boldsymbol{\xi}(t)\rangle^{\text {perturbed }} \equiv\langle\boldsymbol{x}(t)\rangle^{\text {perturbed }}-\langle\boldsymbol{x}(t)\rangle^{\text {unperturbed }} \\
& =\int \boldsymbol{x}\{\hat{p}(\boldsymbol{x} t)-p(\boldsymbol{x} t)\} d x
\end{aligned}
$$

to the external forces,

$$
\langle\xi(t)\rangle^{\text {perturbed }}=\int_{t_{0}}^{t} \chi(t, s) \cdot \boldsymbol{F}(s) d s .
$$

By using Eqs. (3.6) and (3.7), one finds

$\chi(t, s)=\theta(t-s) \int \boldsymbol{x} R(\boldsymbol{x} t \mid \boldsymbol{y} s)[\boldsymbol{\Omega} p(s)]_{\boldsymbol{y}} d x d y$.

We may define a vector-valued state function

$\phi(x t)=[\Omega p(t)]_{x} / p(x t)$ in terms of which the response tensor can be written as a correlation over the unperturbed system,

$\chi(t, s)=\theta(t-s)\langle\boldsymbol{x}(t) \boldsymbol{\phi}(\boldsymbol{x}(s), s)\rangle$.

Since the perturbation cannot change the normalization of the probability density function, one has

$\langle\boldsymbol{\phi}(\boldsymbol{x}(t), t)\rangle \equiv \int[\boldsymbol{\Omega} p(t)]_{x} d x=0$,

i.e. $\phi(t)$ represents a fluctuation (in general nonlinear) from the unperturbed state. Therefore, Eq. (3.12) takes the form of a generalized fluctuation theorem (GFT)

$\chi(t, s)=\theta(t-s)\langle\xi(t) \phi(x(s), s)\rangle$.

An alternative derivation of this result using the technique of functional derivatives is given in the Appendix.

\subsection{Application to Fokker-Planck Processes}

We consider a process which is a solution of the stochastic differential equation [18]

$d \boldsymbol{x}(t)=\boldsymbol{a}(\boldsymbol{x} t) d t+\boldsymbol{b}(\boldsymbol{x} t) \cdot d \boldsymbol{w}(t)$

where $\boldsymbol{w}(t)$ is a vectorial Wiener process with

$\left\langle w_{i}(t)\right\rangle=0, \quad\left\langle\left(w_{i}(t)\right)^{2}\right\rangle=t$.

This process is stochastically equivalent to a vectorial Fokker-Planck process with the stochastic equation

$\frac{\partial}{\partial t} p(\boldsymbol{x} t)=-\boldsymbol{\nabla} \cdot\{\boldsymbol{a}(\boldsymbol{x} t) p(x t)\}+\frac{1}{2}(\boldsymbol{\nabla} \boldsymbol{\nabla}):\{\mathbf{D}(\boldsymbol{x} t) p(\boldsymbol{x} t)\}$

with drift vector $\boldsymbol{a}(\boldsymbol{x} t)$ and diffusion tensor

$\mathbf{D}(x t)=\mathbf{b}(x t) \cdot \tilde{\mathbf{b}}(x t)$.

The perturbations considered are of such a form that a term

$\boldsymbol{F}(t) d t$

is added to the right-hand side of Eq. (3.15). Under this assumption, the perturbation of the stochastic operator also becomes a differential operator,

$\Gamma^{\mathrm{ext}}(t)=-\boldsymbol{F}(t) \cdot \boldsymbol{\nabla}, \quad$ i.e. $\boldsymbol{\Omega}=-\boldsymbol{\nabla}$,

and the function $\phi(x t)$ defined in Eq. (3.11) is given by

$\phi(t)=-\boldsymbol{V} \ln p(t)$.

Therefore, the GFT Eq. (3.14) takes the form

$\chi(t, s)=-\theta(t-s)\langle\xi(t) \boldsymbol{V} \ln p(x(s), s)\rangle$.

In order to obtain an ordinary fluctuation theorem relating the response tensor to the correlation matrix of the fluctuations of the state variables, the function 
$\phi(t)$ must be linear in the fluctuations. This is the case if and only if the unperturbed distribution is a Gaussian,

$p(\boldsymbol{x} t)=\frac{\exp \left\{-\frac{1}{2}(\boldsymbol{x}-\langle\boldsymbol{x}(t)\rangle) \cdot \boldsymbol{\sigma}^{-1}(t) \cdot(\boldsymbol{x}-\langle\boldsymbol{x}(t)\rangle)\right\}}{\{\operatorname{det} 2 \pi \boldsymbol{\sigma}(t)\}^{\frac{1}{2}}}$.

Then,

$\chi(t, s)=\theta(t-s)\langle\xi(t) \xi(s)\rangle \cdot \sigma^{-1}(s)$

or in components

$\chi_{i j}(t, s)=\theta(t-s) \sum_{k} \sigma_{k j}^{-1}(s)\left\langle\xi_{i}(t) \xi_{k}(s)\right\rangle$.

Because of

$\langle\xi(s) \xi(s)\rangle=\sigma(s)$

the response tensor has the property

$\chi(t, s) \rightarrow 1 \quad$ for $t \rightarrow s^{+}$.

A Gaussian distribution is always obtained if the timeevolution operator $R\left(t_{2} \mid t_{1}\right)$ is a Gaussian, and the system is initially prepared in a pure state, $p_{0}(\boldsymbol{x})=$ $\delta\left(\boldsymbol{x}-\boldsymbol{x}_{0}\right)$. Specific examples for such processes are discussed in the next Section.

It should be noted that the derivation of the fluctuation theorem Eq. (3.24) depends only on the form (3.20) of the perturbation operator and the Gaussian form (3.23) of the unperturbed distribution, but not on the unperturbed process being a Fokker-Planck process. This type of process was considered only in order to motivate the form (3.20) of the perturbation operator. Thus, for any Markov process with a perturbation operator (3.20) and a Gaussian distribution there exists a fluctuation theorem of the form (3.24).

\section{Explicit Calculation for a Specific Class of Processes}

We consider the class of one-dimensional processes described by a stochastic differential equation of the form

$d x(t)=-\gamma(t)(x(t)-c(t)) d t+b(t) d w(t)$

equivalent to the "linear" Fokker-Planck equation

$$
\begin{aligned}
& \frac{\partial}{\partial t} p(x, t) \\
& =\frac{\partial}{\partial x}\left\{\gamma(t)(x-c(t)) p(x, t)+\frac{1}{2} D(t) \partial p(x, t) / \partial x\right\} .
\end{aligned}
$$

This class of processes describes systems which relax with a time-dependent rate $\gamma(t) \geqq 0$ to a time-dependent average position $c(t)$, and undergo a diffusion process with a time-dependent diffusion constant $D(t)=$ $b^{2}(t) \geqq 0$.

The time-evolution of such a process is determined by a Gaussian

$R\left(x, t \mid y, t_{1}\right)=\frac{\exp \left\{-\frac{1}{2} \alpha^{-1}\left(t ; t_{1}\right)\left(x-\beta\left(t ; y, t_{1}\right)\right)^{2}\right\}}{\left\{2 \pi \alpha\left(t ; t_{1}\right)\right\}^{\frac{1}{2}}}$

with

$\alpha\left(t ; t_{1}\right)=\int_{t_{1}}^{t} \beta_{1}^{2}(t ; s) D(s) d s$

and

$\beta\left(t ; y, t_{1}\right)=y \beta_{1}\left(t ; t_{1}\right)+\beta_{0}\left(t ; t_{1}\right)$

where

$\beta_{1}\left(t ; t_{1}\right)=\exp \left\{-\left(\Lambda(t)-\Lambda\left(t_{1}\right)\right)\right\}$

$\beta_{0}\left(t ; t_{1}\right)=\int_{t_{1}}^{t} \beta_{1}(t ; s) \gamma(s) c(s) d s$.

Here, $\Lambda(t)$ is given by

$\Lambda(t)=\int_{\text {const }}^{t} \gamma(s) d s$.

This class of processes contains as limiting cases the Gauss-Markov process for $c(t)=0$, yielding

$\beta_{1}\left(t ; t_{1}\right)$ as given in Eq. (4.6)

$\beta_{0}\left(t ; t_{1}\right)=0$;

and the additive Fokker-Planck process for $\gamma(t) \rightarrow 0$, $c(t) \rightarrow \infty$, such that $\gamma(t) c(t) \rightarrow a(t)$, whence

$\beta_{1}\left(t ; t_{1}\right)=1$

$\beta_{0}\left(t ; t_{1}\right)=\int_{t_{1}}^{t} a(s) d s$.

The class of processes considered here has the following simple property: Since $\beta\left(t ; y, t_{1}\right)$ depends linearly on the initial state $y$, an arbitrary initial Gaussian distribution remains Gaussian in its time-evolution. As a consequence, a fluctuation theorem (3.24) holds for all systems prepared with an initial Gaussian distribution. For the fluctuation $\langle\xi(t) \xi(s)\rangle$ one obtains the simple result

$\langle\xi(t) \xi(s)\rangle=\beta_{1}(t, s) \sigma(s)$

where $\sigma^{\frac{1}{2}}(s)$ is the width of the distribution at time $s$. Therefore,

$\chi(t, s)=\beta_{1}(t, s) \theta(t-s)$.

The response has thus the remarkable property that it is independent of the initial distribution as long as the latter is a Gaussian. This is a direct consequence 
of the linearity of $\beta\left(t ; t_{1}, y\right)$ with $y$. The response is also independent of $c(t)$ and $D(t)$, and is completely determined by the function $\gamma(t)$.

If the process is ergodic, the fluctuation theorem (3.24) holds also asymptotically, independent of the initial distribution being Gaussian. In order to study the question of ergodicity one has to note that $\Lambda(t)$ is a monotonously increasing function of time. There are two possibilities:

a) If $\Lambda\left(\mathrm{t}_{0}\right) \rightarrow-\infty$, i.e. $\beta_{1}\left(t, t_{0}\right) \rightarrow 0$, for $t_{0} \rightarrow-\infty$, then the process is ergodic if

$\alpha(t)=\int_{-\infty}^{t} \beta_{1}^{2}(t, s) D(s) d s$

and

$\beta(t)=\int_{-\infty}^{t} \beta_{1}(t, s) \gamma(s) c(s) d s$

exist. The asymptotic distribution is then given by

$p^{\text {as }}(t)=\{2 \pi \alpha(t)\}^{-\frac{1}{2}} \exp \left\{-\frac{1}{2} \alpha^{-1}(t)(x-\beta(t))^{2}\right\}$.

b) If $A\left(t_{0}\right) \rightarrow$ const for $t_{0} \rightarrow-\infty$, then $\beta_{1}(t ; y,-\infty)$ depends on the initial state $y$, and the process is nonergodic. This is in particular true for the additive Fokker-Planck process.

\section{Discussion}

We have derived a generalized fluctuation theorem for nonstationary Markov processes which reduces to an ordinary fluctuation theorem for the class of "linear" Fokker-Planck processes. There exist numerous examples for such type of process; we mention only two applications: The charge on the capacitor of an $R C$-circuit with a time-dependent capacitance or resistance (as in a microphone) is a nonstationary Fokker-Planck process with $\gamma(t)=1 /(R(t) C(t))$. The concentration $c(t)$ of one component of a chemical reaction with an equilibrium at $c_{0}(T)$ and a reaction rate $r(T)$ becomes a nonstationary process if the reaction takes place at a time-dependent temperature $T(t)$.

We add some comments concerning the significance of the response function $\chi\left(t_{2}, t_{1}\right)$ and the autocorrelation function $S\left(t_{2}, t_{1}\right)=\left\langle\xi\left(t_{2}\right) \xi\left(t_{1}\right)\right\rangle$ of a nonstationary process from an experimental point of view. The response function $\chi\left(t_{2}, t_{1}\right)$ can in principle be measured as the response at time $t_{2}$ to a $\delta$-impulse at time $t_{1}$, and its double Fourier transform [15] $\chi\left(\omega_{2}, \omega_{1}\right)$ can be measured as the response at frequency $\omega_{2}$ to a harmonic force at frequency $\omega_{1}$. In contrast to the stationary case, $\chi\left(t_{2}, t_{1}\right)$ depends on $t_{1}$ and $t_{2}$ separately, and as a consequence, one obtains a response also at frequencies $\omega_{2} \neq \omega_{1}$. The double Fourier transform $S\left(\omega_{2}, \omega_{1}\right)$ of the autocorrelation function can be measured as the correlation between Fourier components $\xi\left(\omega_{2}\right)$ and $\xi\left(\omega_{1}\right)$ of the fluctuations. Whereas in the stationary case, fluctuations at different frequencies are uncorrelated, and only the meansquare amplitude ("power spectrum")

$S(\omega)=\left\langle|\xi(\omega)|^{2}\right\rangle \Delta \omega$

remains, in the nonstationary case there exists a correlation also for different frequencies.

From the causality requirement expressed by the $\theta$ function in Eq. (3.10), there follow dispersion relations for the double Fourier transform $\chi\left(\omega_{2}, \omega_{1}\right)$ also in the nonstationary case. At fixed $\omega_{1}, \chi\left(\omega_{2}, \omega_{1}\right)$ can be analytically continued into the complex $\omega_{2}$-plane, and is analytic in the upper half plane. Similarly, at fixed $\omega_{2}, \chi\left(\omega_{2}, \omega_{1}\right)$ can be continued into the complex $\omega_{1}$ plane, and is analytic in the lower half plane. As a consequence, one finds dispersion relations for both variables separately,

$\chi^{\prime}\left(\omega_{2}, \omega_{1}\right)=\frac{1}{\pi} \int_{-\infty}^{+\infty} \frac{\chi^{\prime \prime}\left(x, \omega_{1}\right)}{x-\omega_{2}} d x=\frac{1}{\pi} f_{-\infty}^{+\infty} \frac{\chi^{\prime \prime}\left(\omega_{2}, x\right)}{\omega_{1}-x} d x$

$\chi^{\prime \prime}\left(\omega_{2}, \omega_{1}\right)=-\frac{1}{\pi} f_{-\infty}^{+\infty} \frac{\chi^{\prime}\left(x, \omega_{1}\right)}{x-\omega_{2}} d x=-\frac{1}{\pi} f_{-\infty}^{+\infty} \frac{\chi^{\prime}\left(\omega_{2}, x\right)}{\omega_{1}-x} d x$.

Of practical use is also the generalization of the Wiener-Khintchin theorem for nonstationary processes [16].

\section{Appendix}

We give here an alternative derivation of the linear response formalism based on functional derivates. The response tensor defined by Eq. (3.9) can be written as

$\boldsymbol{\chi}(t, s)=\delta\langle\boldsymbol{x}(t)\rangle^{\text {perturbed }} / \delta \boldsymbol{F}(s)$

$=\int \boldsymbol{x}\{\delta \hat{p}(\boldsymbol{x} t) / \delta \boldsymbol{F}(s)\} d x$,

where the functional derivatives are taken at $\boldsymbol{F}(s)=0$. Now, from the linearized equation of motion for the perturbed distribution,

$\dot{\hat{p}}(t)=\Gamma(t) \hat{p}(t)+\boldsymbol{F}(t) \cdot \boldsymbol{\Omega} p(t)$,

one obtains on account of $\delta \boldsymbol{F}(t) / \delta \boldsymbol{F}(s)=\mathbf{1} \delta(t-s)$

$d / d t\{\delta \hat{p}(t) / \delta \boldsymbol{F}(s)\}$

$=\Gamma(t)\{\delta \hat{p}(t) / \delta \boldsymbol{F}(s)\}+\boldsymbol{\Omega} p(s) \delta(t-s)$.

This is a differential equation for $\delta \hat{p}(t) / \delta \boldsymbol{F}(s)$ with the 
solution

$\delta \hat{p}(t) / \delta \boldsymbol{F}(s)=R(t \mid s) \boldsymbol{\Omega} p(s) \theta(t-s)$.

This result suggests the following simple interpretation for the linear response of the distribution function to the perturbation $\delta \boldsymbol{F}(s)=\boldsymbol{F}(s) d s$ : The distribution gets an impulse of strength $\delta \boldsymbol{F}(s) \cdot \boldsymbol{\Omega} p(s)$ at time $s$ which then evolves according to the unperturbed timeevolution $R(t \mid s)$.

Substitution of Eq. (A.4) into Eq. (A.1) leads to Eq. (3.10) found in Section 3.

\section{References}

1. For a detailed review of the extended literature see Haken, $\mathrm{H}$.: Rev. Mod. Phys. 47, 67 (1975)

2. Landau, L.D., Lifschitz, E. M.: Statistical Physics, Vol. V, chapt. XII. London-Paris: Pergamon Press 1958

3. Kubo, R.: Rep. Prog. Phys. 29, 255 (1966)

4. De Groot, S. R., Mazur, P.: Nonequilibrium Thermodynamies, chapt. VIII. Amsterdam: North-Holland 1962

5. Stahl, A., Bausch, R., Janssen, H.K.: Phys. Letters 24 A, 171 (1967)
6. Weidlich, W.: Z. Physik 248, 234 (1971)

7. Agarwal, G. S.: Z. Physik 252, 25 (1972)

8. Agarwal, G. S.: Phys. Letters 38 A, 93 (1972)

9. Lindenberg, K.L., Bedeaux, D. : Physica 57, 157 (1972)

10. Decker, U., Haake, H.: Phys. Rev. 11 A, 2043 (1975)

11. Lax, M.: Rev. Mod. Phys. 38, 371 (1966)

12. Takács, L.: Stochastic Processes: Problems and Solutions, chapt. II. London: Methuen (1960)

13. Doob, J. L.: Stochastic Processes, chapt. VI. New York: Wiley 1953

14. Cox, D.R., Miller, H.D.: The Theory of Stochastic Processes, chapt. IV. London: Methuen 1965

15. Papoulis, A.: Probability, Random Variables and Stochastic Processes, chapt. XII. New York: McGraw-Hill 1965

16. Kouskoulas, V.: J. Appl. Phys. 42, 3659 (1971)

17. Feynman, R. P.: Phys. Rev. 84, 108 (1951)

18. Gihman, I. I., Skorohod, A.V.: Stochastic Differential Equations, ed. by Halmos, P.R. et al. Berlin-Heidelberg-New York: Springer 1972

Dipl.-Phys. Peter Hänggi

Prof. Dr. Harry Thomas

Institut für Physik der Universität Basel

Klingelbergstrasse 82

CH-4056 Basel

Switzerland 